\title{
Differentiation between Benign and Malignant Breast Lesions Using ADC on Diffusion-Weighted Imaging at 3.0 T
}

\author{
Vikash Kr Gupta, Wanhua Liu*, Rui Wang, Yuanyuan Ye, Jing Jiang \\ Department of Radiology, School of medicine, Zhongda Hospital, Southeast University, Nanjing, China \\ Email: *liuwanhua.com@126.com
}

Received 25 November 2015; accepted 5 March 2016; published 9 March 2016

Copyright (C) 2016 by authors and Scientific Research Publishing Inc.

This work is licensed under the Creative Commons Attribution International License (CC BY). http://creativecommons.org/licenses/by/4.0/

(c) (i) Open Access

\section{Abstract}

Aim: To investigate the diagnostic efficiency of apparent diffusion coefficient value (ADC) in differentiating benign from malignant breast lesions at 3.0 $\mathrm{T}$ diffusion-weighted imaging with different pair of b value. Methods: Total 110 patients with 107 lesions (44 benign and 63 malignant) were selected for our study with five different $b$-values $0,400,800,1200$ and $1600 \mathrm{~s} / \mathrm{mm}^{2}$. ADC values were calculated using different pairs of $b$ values. The cut-off ADC values and diagnostic efficiency were evaluated by receiver operating characteristic analysis. Comparison of Mean ADC value for breast lesions was determined by using independent sample $t$ test. ROC curves were used for diagnostic efficiency of ADC using different pairs of $b$ values. Results: With increase of $b$ value, mean ADC value decreases. The mean ADC values for benign were $1.73 \times 10^{-3} \mathrm{~mm}^{2} / \mathrm{s}$ for $\mathrm{b} 0$ and $400,1.57 \times 10^{-3}$ $\mathrm{mm}^{2} / \mathrm{s}$ for $b 0$ and $800,1.43 \times 10^{-3} \mathrm{~mm}^{2} / \mathrm{s}$ for $b 0$ and 1200 and $1.30 \times 10^{-3} \mathrm{~mm}^{2} / \mathrm{s}$ for $\mathrm{b} 0$ and 1600 $\mathrm{s} / \mathrm{mm}^{2}$. The mean ADC values for the malignant breast lesion were $1.21 \times 10^{-3} \mathrm{~mm}^{2} / \mathrm{s}$ for $\mathrm{b} 0$ and $400,1.06 \times 10^{-3} \mathrm{~mm}^{2} / \mathrm{s}$ for $b 0$ and $800,0.94 \times 10^{-3} \mathrm{~mm}^{2} / \mathrm{s}$ for $b 0$ and 1200 and $0.86 \times 10^{-3} \mathrm{~mm}^{2} / \mathrm{s}$ for $b 0$ and $1600 \mathrm{~s} / \mathrm{mm}^{2}$. ADC diagnostic efficiency for benign and malignant lesion for all the pair of $b$ value combination was significant $(p>0.05)$. The sensitivity, specificity, PPV, NPV and accuracy were $80.95 \%, 90.9 \%, 92.72 \%, 76.92 \%, 85.04 \%$ for b 0 and $400 ; 84.12 \%, 90.9 \%, 92.98 \%, 80 \%$, $86.91 \%$ for $b$ and $800 ; 84.12 \%, 90.9 \%, 92.98 \%, 80 \%, 86.91 \%$ for $b 0$ and $1200 ; 84.12 \%, 90.9 \%$, $92.98 \%, 80 \%, 86.91 \%$ for $b 0$ and $1600 \mathrm{~s} / \mathrm{mm}^{2}$ respectively. Conclusion: DWI is effective in differentiating benign and malignant breast lesion at 3.0 Tesla using ADC with higher $b$ value combination.

\section{Keywords}

Diffusion-Weighted Magnetic Resonance Imaging, b-Values, Apparent Diffusion Coefficient, Breast Lesion

\footnotetext{
${ }^{*}$ Corresponding author.
}

How to cite this paper: Gupta, V.K., Liu, W.H., Wang, R., Ye, Y.Y. and Jiang, J. (2016) Differentiation between Benign and Malignant Breast Lesions Using ADC on Diffusion-Weighted Imaging at 3.0 T. Open Journal of Radiology, 6, 1-9. 


\section{Introduction}

One of the most common types of tumor affecting female nowadays is breast cancer. Annually, over 1,000,000 new cases are diagnosed worldwide [1]. So to reduce mortality rates caused due to breast cancer we need early diagnosis and treatment to increase survival and improve the quality of life, hence diagnostic imaging is necessary with accuracy. Over the past few years, MRI has been used to study breast lesions [2]-[4]. Magnetic resonance imaging is a widely accepted modality as an adjunct to mammography and ultrasonography in the detection of primary or recurrent breast cancer [5]. Hence, several studies have been investigating the role of advanced MRI techniques, such as diffusion-weighted imaging (DWI), to improve the specificity of diffusion weighted MRI for the evaluation of breast carcinoma [6]-[10]. DWI measures the random movement of water molecules in tissues, which is detected as the attenuation of the measured signal intensity [11] on Diffusion weighted MRI (DWI). Diffusion can be quantified by using the apparent diffusion coefficient (ADC) value to increase the specificity of DWI [12]. The movement of water molecules is much more restricted in tissues with a higher cellular content and associated with numerous intact cell membranes [13] [14] as seen in malignant breast lesion. The cell density is increased in malignant breast cancer, which corresponds to lower ADC values [13] [15]-[17] on DWI.

While the sensitivity of dynamic contrast-enhanced (DCE) MRI in the diagnosis of breast cancer is approximately $85 \%$ - 99\%, its specificity ranges between $40 \%$ and $80 \%$ as stated by Kuhl, C.K. et al. [18]. Diffusionweighted imaging (DWI) is the techniques that are performed to increase MRI specificity [7] [8]. The sensitivity of MRI for breast cancer detection ranges from $85 \%$ - 100\% [19] [20], and specificity ranges from only $37 \%$ 88\% [21] [22]. DWI-MRI is used to detect cancers of the body easily as it has shown that diffusion of the water molecules in malignant tumors is slower compared to the normal body parenchyma.

The purpose of this study is to investigate the diagnostic efficiency of the apparent diffusion coefficient value (ADC) in differentiating benign from malignant breast lesions at 3.0 T Diffusion weighted MRI with different pairs of $b$ value.

\section{Materials and Methods}

\subsection{Subjects}

A retrospective clinical breast DWI MRI examination was reviewed from March 2011 to April 2015 in our hospital. The indication for MR imaging were mainly women suspected of breast cancer, high risk women, preoperative local staging, follow up after conservative breast surgery, detection of unknown primary cancer and women suspected of breast lump in conventional mammography or ultrasonography. This study was approved by the institutional review board, and informed written consent was obtained from all patients after the procedure had been explained. Patients enrolled into this study had diagnostic procedure, including the following: a) 3.0T DW-MRI done prior to surgery b) histopathological confirmed diagnosis after surgery c) Good-quality DW MR scans images. Exclusion criteria were 1) Patient with inflammation and cysts 2) Patient with neo-adjuvant chemotherapy 3) those with poor DWI or DCE-MRI image quality 4) those with breast implants 5) those who fail to fat suppression were excluded from the study as it could give us false readings.

On the basis of these criteria, we excluded eight lesions in eight patients (two were breast cyst, three were due to chemotherapy, two due to poor image quality and one due to breast implants). As a result, the study included a total of 110 women with 107 lesions (mean age 46.09 years; range 25 - 68 years) diagnosed with benign and malignant breast lesion constituted our study group. The mean size of benign lesions was $1.9 \mathrm{~cm}$ (range $0.9 \mathrm{~cm}$ $4.2 \mathrm{~cm}$ ), while that of malignant lesions was $4.5 \mathrm{~cm}$ (range $1.0 \mathrm{~cm}-10.2 \mathrm{~cm}$ ). Of the 107 lesion 44 were benign and 63 were malignant lesion. Among the 44 benign included 22 fibroadenoma, 18 adenosis, 2 papilloma, 1 lobulated tumor and 1 scleorsing adenosis and among 63 malignant tumor included 37 IDC (Invasive ductal Carcinoma), 12 DCIS (Ductal Carcinoma in situ), 9 Invasive lobular carcinoma(ILC), 5 mucinous carcinoma, 2 medullary carcinoma, 1 squamous cell carcinoma and 1 papillary carcinoma.

\subsection{MRI Protocol}

All DWI-MRI was performed on 3.0-T MR System (Magnetom Verio, Siemens, Germany) by using a dedicated bilateral breast coil with patients in prone position. Before DWI, standard sequences were acquired, including an axial fat-suppressed T2-weighted images turbo inversion recovery magnitude (TIRM; TR/TE,4300/61; inversion time, 150 milliseconds; bandwidth, $319 \mathrm{~Hz} / \mathrm{Px}$; matrix size, $320 \times 320$; field of view, $340 \mathrm{~mm}$; number of sig- 
nals averaged, 2; slice thickness, 4 mm;), axial T1-weighted spin-echo sequence (TR/TE, 6.04/2.45; bandwidth, $400 \mathrm{~Hz} / \mathrm{Px}$; matrix size, $512 \times 512$; field of view, $340 \mathrm{~mm}$; number of signals averaged, 1; slice thickness, 1.3 $\mathrm{mm}$;), axial T2-weighted spin-echo sequence (TR/TE, 1000/87; bandwidth, $244 \mathrm{~Hz} / \mathrm{Px}$; matrix size, $512 \times 512$; field of view, $340 \mathrm{~mm}$; number of signals averaged, 1; slice thickness, $2.0 \mathrm{~mm}$ ), and axial T1-weighted 3D fat-suppressed fast spoiled gradient-echo sequence (TR/TE, 4.67/1.66; bandwidth, $320 \mathrm{~Hz} / \mathrm{Px}$; matrix size, $384 \times$ 384; field of view, $360 \mathrm{~mm}$; slice thickness, $1.20 \mathrm{~mm}$ ) before and eight times after the rapid injection of a bolus of intravenous contrast medium (gadopentetate dimeglumine) at a dose of $0.2 \mathrm{mmol} / \mathrm{kg}$ body weight infused at the rate of $2 \mathrm{ml} / \mathrm{sec}$, followed by $20 \mathrm{ml}$ of saline solution. After the examination, the unenhanced images were subtracted from the first and last contrast-enhanced images.

DCE-MRI images were obtained with a T1W 3D FLASH sequence using spectrally adiabatic inversion recovery (SPAIR) for fat suppression. DWI was performed before the DCE-MRI acquisition in an axial plane using a 2D spin echo-echo planar imaging (SE-EPI) sequence centered on the lesion acquired in 7 minutes 21 seconds for the DWI scan $\left(b=0,400,800,1200\right.$, and $1600 \mathrm{~s} / \mathrm{mm}^{2}$; repetition time (TR)/time to echo (TE): 10,500/87 ms; bandwidth, $1184 \mathrm{~Hz} / \mathrm{Px}$; matrix size, $192 \times 192$; field of view, $350 \mathrm{~mm}$; number of slices, 26; slice thickness, $4 \mathrm{~mm}$; number of signal averaged, 3; PAT mode GRAPPA; acceleration factor PE, 2; total scan time 7:21sec.

\subsection{Analysis of MR Images}

All images were transferred to a workstation (Magnetom Verio, Siemens) and the DWI sequence was post processed with the software (Magnetom Verio, Siemens) to obtain ADC maps (black-and-white). Two researchers retrospectively analyzed the diffusion-weighted (DW) Images and at the time of DWI analysis, readers were unaware of histological results. The ADC maps of each lesion were calculated using two b values ( 0 and 400,0 and 800, 0 and 1200, 0 and $1600 \mathrm{~s} / \mathrm{mm}^{2}$ ). The dynamic contrast enhancement MR (DCE-MR) was used as the reference to locate the lesion. To achieve standardized conditions for analyses and to avoid contamination of the data by adjacent structures, three circular regions of interest (ROIs) were drawn manually in the central region of the homogeneous breast lesions in the same location as the five DW imaging and the four ADC maps cited above, and the average ADC value was recorded for each b value combination. Apparent necrotic, cystic or hemorrhagic components were avoided by referring to conventional MR images.

The ROI sizes were set to a mean diameter of $50 \mathrm{~mm}^{2}$ for breast lesions and the mean value from 3 ROIs were recorded for ADC.

\subsection{Statistical Analysis}

Data were analyzed using the IBM SPSS 21.0 software package (SPSS Inc, Chicago, IL, USA). All Quantitative data were expressed by mean \pm standard deviation. Mean ADC value for benign and malignant breast lesions were calculated. Differences in mean ADC between benign and malignant breast lesion were calculated using independent sample t-test. Receiver operating characteristic (ROC) analysis was performed to determine the best cut off score and area under curve (AUC) for each pair of b value using ADC, so that benign and malignant lesions could be differentiated. ROC curve analysis was also used to determine the diagnostic efficiency of the ADC to calculate the sensitivity, specificity, PPV, NPV and accuracy rate. Statistical significance was set at $\mathrm{p}<0.05$.

\section{Results}

\subsection{Comparison of the ADC Values of Benign and Malignant Lesions for Different Pairs of b-Values}

Table 1 illustrates the mean ADC values for benign and malignant breast lesions for all the pairs of $b$ value ( 0 400, $0-800,0-1200,0-1600 \mathrm{~s} / \mathrm{mm}^{2}$ ) and the results shows that there is significant differences between the benign and malignant breast lesions $(\mathrm{p}<0.05)$. The mean ADC value for benign were found to be $1.73 \times 10^{-3}$ $\mathrm{mm}^{2} / \mathrm{s}$ for b0 and $400,1.57 \times 10^{-3} \mathrm{~mm}^{2} / \mathrm{s}$ for b0 and $800,1.43 \times 10^{-3} \mathrm{~mm}^{2} / \mathrm{s}$ for b 0 and 1200 and $1.30 \times 10^{-3}$ $\mathrm{mm}^{2} / \mathrm{s}$ for b0 and $1600 \mathrm{~s} / \mathrm{mm}^{2}$. The mean ADC value for the malignant breast lesion were found to be $1.21 \times$ $10^{-3} \mathrm{~mm}^{2} / \mathrm{s}$ for b0 and $400,1.06 \times 10^{-3} \mathrm{~mm}^{2} / \mathrm{s}$ for b0 and $800,0.94 \times 10^{-3} \mathrm{~mm}^{2} / \mathrm{s}$ for b0 and 1200 and $0.86 \times$ $10^{-3} \mathrm{~mm}^{2} / \mathrm{s}$ for b0 and $1600 \mathrm{~s} / \mathrm{mm}^{2}$. The results shows that the mean ADC values for the malignant breast lesion had lower ADC value compared to benign breast lesion for all $b$ values in pair as shown in Figure 1, and the mean ADC value decreases with the increase in $b$ value pair for both the benign and malignant breast lesion. 


\subsection{Diagnostic Efficiency of ADC Value Using ROC Curve for Different Pairs of b-Values}

ROC (Receiver Operating Characteristics) curves for all the pair of b values are represented in Figure 2. The

Table 1. Comparison of the ADC values for benign and malignant breast lesions.

\begin{tabular}{|c|c|c|c|}
\hline \multirow{2}{*}{ b- values combination } & \multicolumn{2}{|c|}{ ADC $\left(\times 10^{-3} \mathrm{~mm}^{2} / \mathrm{s}\right)$} & \multirow{2}{*}{$p$-value } \\
\hline & Benign & Malignant & \\
\hline $0-400 \mathrm{~s} / \mathrm{mm}^{2}$ & $1.73 \pm 0.31$ & $1.21 \pm 0.40$ & $0.00^{* *}$ \\
\hline $0-800 \mathrm{~s} / \mathrm{mm}^{2}$ & $1.57 \pm 0.32$ & $1.06 \pm 0.38$ & $0.00^{* *}$ \\
\hline $0-1200 \mathrm{~s} / \mathrm{mm}^{2}$ & $1.43 \pm 0.30$ & $0.94 \pm 0.36$ & $0.00^{* *}$ \\
\hline $0-1600 \mathrm{~s} / \mathrm{mm}^{2}$ & $1.30 \pm 0.29$ & $0.86 \pm 0.33$ & $0.00^{* *}$ \\
\hline
\end{tabular}

Note: ${ }^{*}$ Quantitative data represented as Mean \pm S.D. ${ }^{* *} p$-value shows statistically significant $(\mathrm{p}<0.05)$.

ADC: apparent diffusion coefficient; SD: standard deviation.

\section{Comparison of ADC benign and malignant breast lesion}

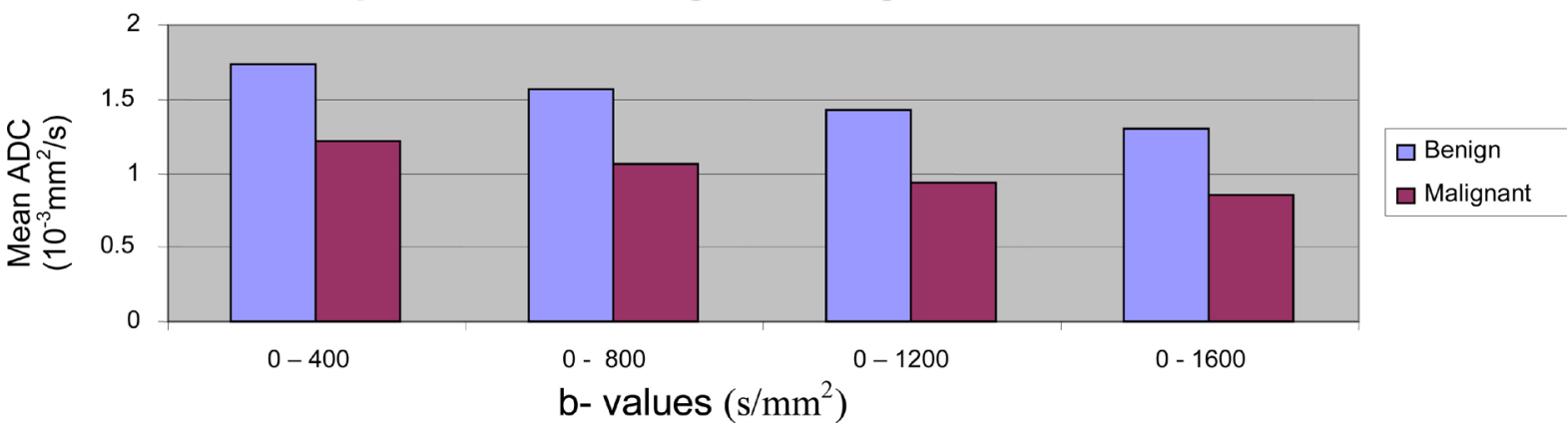

Figure 1. Mean ADC value at 3.0 Tesla using different pairs of b value in differentiating benign and malignant breast lesion. The Mean ADC value is indicated along the y-axis. The mean ADC for malignant breast lesions are significantly lower than benign breast lesion for all pair of b-value $\left(0-400,0-800,0-1200,0-1600 \mathrm{~s} / \mathrm{mm}^{2}\right)$.

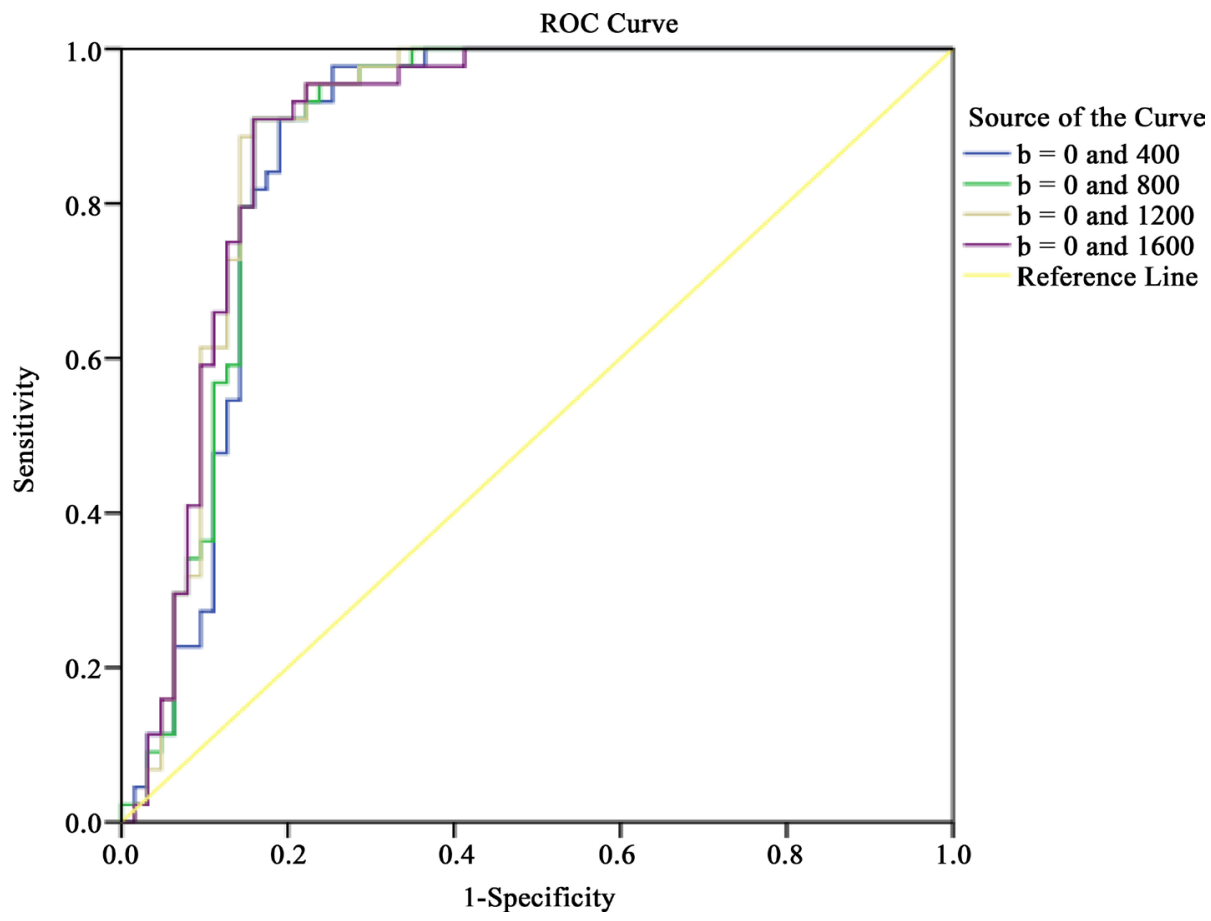

Figure 2. ROC curves. Receiver operating characteristic (ROC) curves derived from Apparent Diffusion Co-efficient (ADC) in differentiating malignant from benign lesions at 3.0 Tesla DWI. The areas under the curves (AUC) at b values of 0 - 400 , $0-800,0-1200$ and $0-1600 \mathrm{~s} / \mathrm{mm}^{2}$ were $0.87,0.88,0.89$, and 0.89 respectively. 
ROC diagnostic efficiency of ADC for different b value pair were statistically significant in differentiation of benign and malignant breast lesion ( $p=0.00$ ) (Figure 3, Figure 4). The cut-off ADC value was found to be 1.35 $\times 10^{-3} \mathrm{~mm}^{2} / \mathrm{s}$ for b 0 and $400,1.22 \times 10^{-3} \mathrm{~mm}^{2} / \mathrm{s}$ for b 0 and $800,1.08 \times 10^{-3} \mathrm{~mm}^{2} / \mathrm{s}$ for b 0 and 1200 and $0.99 \times$ $10^{-3} \mathrm{~mm}^{2} / \mathrm{s}$ for b0 and $1600 \mathrm{~s} / \mathrm{mm}^{2}$ as demonstrated in Table 2. The mean ADC value, which was equal or less than the cut-off value, suggested malignant breast cancer. The Sensitivity, Specificity, PPV, NPV and accuracy were $80.95 \%, 90.9 \%, 92.72 \%, 76.92 \%, 85.04 \%$ for b 0 and $400 ; 84.12 \%, 90.9 \%, 92.98 \%, 80 \%, 86.91 \%$ for b0 and 800 ; $84.12 \%$, 90.9\%, 92.98\%, 80\%, 86.91\% for b0 and 1200 ; $84.12 \%, 90.9 \%, 92.98 \%$, $80 \%, 86.91 \%$ for b0 and $1600 \mathrm{~s} / \mathrm{mm}^{2}$ respectively. Significant result were obtained for all the $b$ value combination $(\mathrm{p}<0.05)$ as shown in Table 2 obtained from ROC curves analysis using ADC values in differentiating benign and malignant breast lesion at 3.0 Tesla DW-MRI.

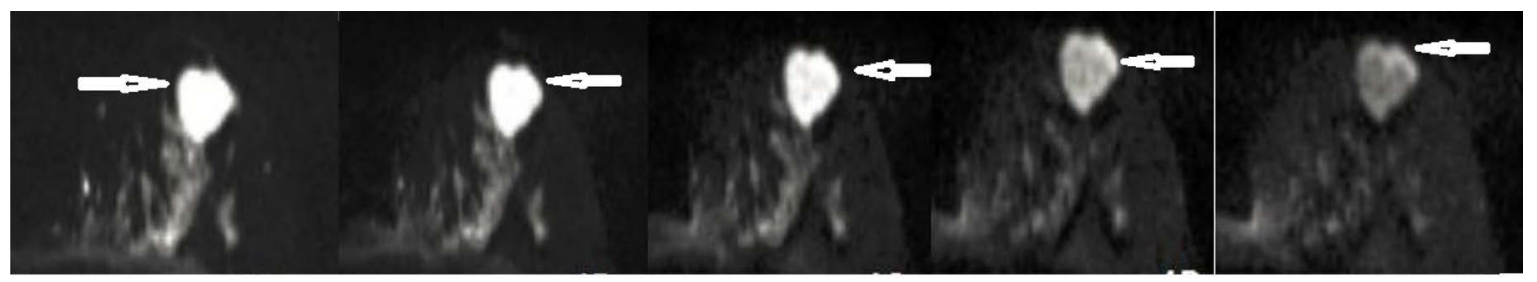

(a) (c) (d) (e)

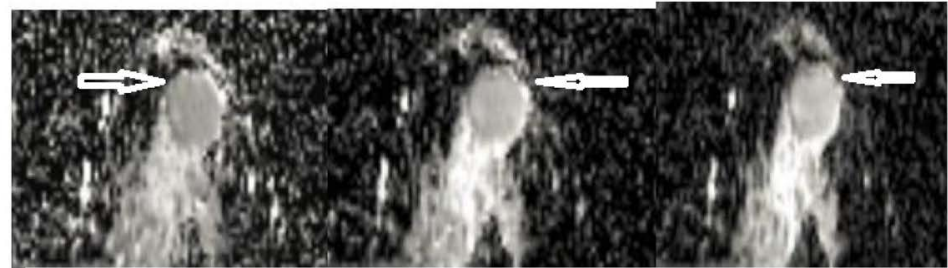

(f) (g) (h)

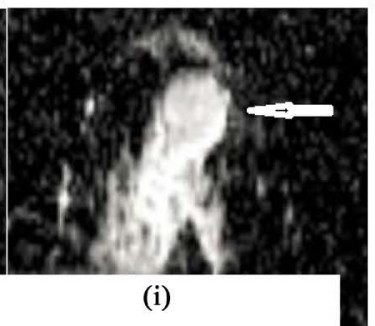

Figure 3. 44 year female, fibroadenoma left breast. (a)-(e), DWI with b-value of $0 \mathrm{~s} / \mathrm{mm}^{2}, 400 \mathrm{~s} / \mathrm{mm}^{2}, 800 \mathrm{~s} / \mathrm{mm}^{2}, 1200$ $\mathrm{s} / \mathrm{mm}^{2}, 1600 \mathrm{~s} / \mathrm{mm}^{2}$ reveals homogeneous high signal intensity in the left breast (arrow) (f)-(i), Apparent diffusion coefficient (ADC) map with b-value of $400 \mathrm{~s} / \mathrm{mm}^{2}, 800 \mathrm{~s} / \mathrm{mm}^{2}, 1200 \mathrm{~s} / \mathrm{mm}^{2}, 1600 \mathrm{~s} / \mathrm{mm}^{2}$ shows hypo intensity lesion (arrow). The signal intensity of the benign lesion was significantly higher than the normal glandular tissue, meanwhile with the increasing of b-value the signal intensity decreased and the declining degree of signal intensity of benign breast lesion is higher than that of malignant lesions shown in Figure 4 for each of the b-values.

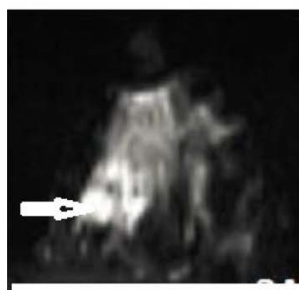

(a)

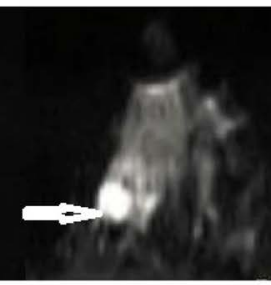

(b)

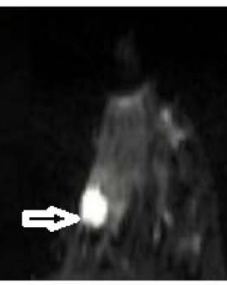

(c)

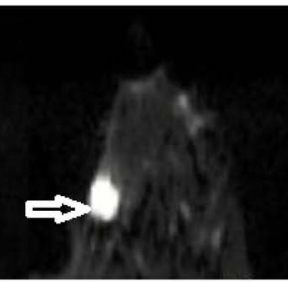

(d)

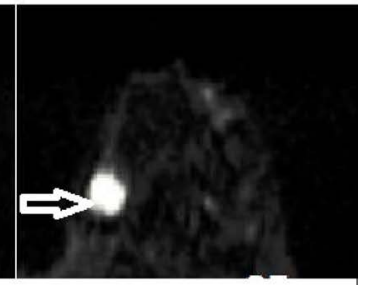

(e)

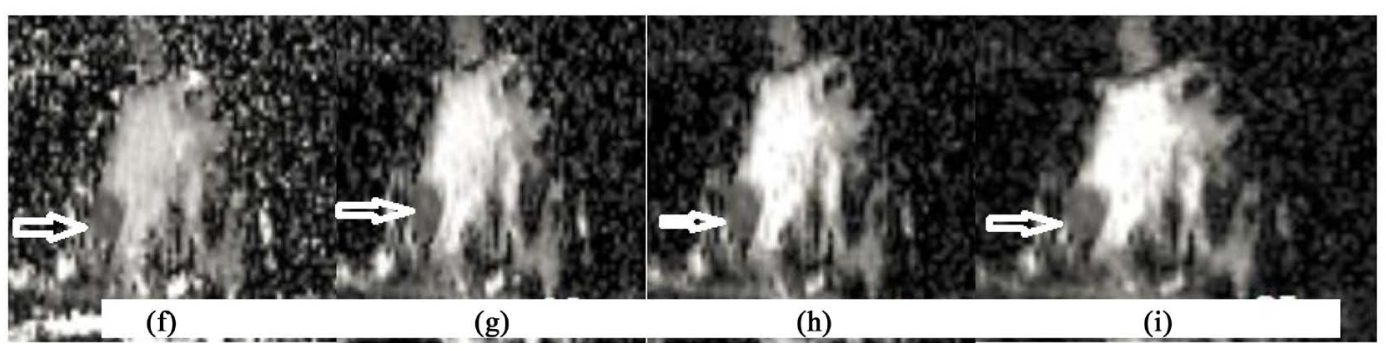

Figure 4. A 41-year-old woman with invasive ductal carcinoma (IDC) left breast. (a)-(e), DWI with b-value of $0 \mathrm{~s} / \mathrm{mm}^{2}, 400$ $\mathrm{s} / \mathrm{mm}^{2}, 800 \mathrm{~s} / \mathrm{mm}^{2}, 1200 \mathrm{~s} / \mathrm{mm}^{2}, 1600 \mathrm{~s} / \mathrm{mm}^{2}$ reveals homogeneous high signal intensity in the left breast (arrow) (f)-(i), Apparent diffusion coefficient (ADC) map with b-value of $400 \mathrm{~s} / \mathrm{mm}^{2}, 800 \mathrm{~s} / \mathrm{mm}^{2}, 1200 \mathrm{~s} / \mathrm{mm}^{2}, 1600 \mathrm{~s} / \mathrm{mm}^{2}$ shows low signal intensity lesion as restricted diffusion (arrow). 
Table 2. Diagnostic efficiency of ADC in differentiation of breast lesion using ROC curves.

\begin{tabular}{|c|c|c|c|c|}
\hline \multirow{2}{*}{ Parameter } & \multicolumn{4}{|c|}{ b-Value Combination $\left(\mathrm{s} / \mathrm{mm}^{2}\right)$} \\
\hline & $0-400 \mathrm{~s} / \mathrm{mm}^{2}$ & $0-800 \mathrm{~s} / \mathrm{mm}^{2}$ & $0-1200 \mathrm{~s} / \mathrm{mm}^{2}$ & $0-1600 \mathrm{~s} / \mathrm{mm}^{2}$ \\
\hline AUC $^{*}$ & 0.87 & 0.88 & 0.89 & 0.89 \\
\hline Cut off Score $\left(\times 10^{-3} \mathrm{~mm}^{2} / \mathrm{s}\right)$ & 1.35 & 1.22 & 1.08 & 0.99 \\
\hline Sensitivity (\%) & 80.95 & 84.12 & 84.12 & 84.12 \\
\hline Specificity (\%) & 90.9 & 90.9 & 90.9 & 90.9 \\
\hline PPV (\%) & 92.72 & 92.98 & 92.98 & 92.98 \\
\hline NPV (\%) & 76.92 & 80 & 80 & 80 \\
\hline ACC (\%) & 85.04 & 86.91 & 86.91 & 86.91 \\
\hline$p-$ Value $^{* *}$ & $0.00^{* *}$ & $0.00^{* *}$ & $0.00^{* *}$ & $0.00^{* *}$ \\
\hline
\end{tabular}

Note: ${ }^{*}$ AUC: Area under Curve from ROC curves. ${ }^{* *} p$ value shows statistically significant $(\mathrm{p}<0.05)$.

$\mathrm{PPV}=$ Positive predictive value; NPV = Negative predictive value; ACC = Accuracy Rate.

\section{Discussion}

Water diffusion in body tissues is the process that reflects the exchange of different components between the extra cellular (ECF) and intracellular (ICF) fluid compartment and the interstitial fluid in human body. In normal human tissues water movement is affected by the cell membranes and macromolecules within the cell. Diffusion Weighted Imaging (DWI) shows the random motion of water molecules and is the only method of measuring molecule diffusion [23]. Many research shows that the breast lesions may be differentiated by calculating the mean ADC values, with malignant cancer yielding lower ADC values compared to benign or normal tissues [24]-[26].

In our study we tested the diagnostic efficiency of ADC in differentiating benign and malignant breast lesion. The mean ADC value for the malignant breast lesion was significantly lower than the benign lesion for all the combination of $b$ values used in our study. This finding was as same as that of the literature stated by [16] [24] [27]-[34]. We also noted that with the higher $b$ values combination the ADC value decreases. However, statistical differences $(\mathrm{p}<0.05)$ was seen for all the mean ADC values calculated from different $b$ values combination in our study. Many studies related to DWI have shown results in differentiating benign and malignant breast lesions with sensitivity ranging from $81 \%$ to $93 \%$ and specificity from $80 \%$ to $88.5 \%$ [13] [15] [24] [26] [35]-[37]. Our results corroborate with those previous studies and the calculated ADC values from different $b$ values combinations and all of them were statically significant.

We found that diffusion restriction in a lesion as a bright signal (high intensity) on DWI and as dark signal (low intensity) on corresponding ADC map, having a Sensitivity, Specificity, PPV, NPV and accuracy rate were 80.95\%, 90.9\%, 92.72\%, 76.92\%, 85.04\% for b0 and 400; 84.12\%, 90.9\%, 92.98\%, 80\%, $86.91 \%$ for b0 and 800; $84.12 \%, 90.9 \%, 92.98 \%, 80 \%, 86.91 \%$ for b0 and 1200 ; $84.12 \%, 90.9 \%, 92.98 \%, 80 \%, 86.91 \%$ for b0 and $1600 \mathrm{~s} / \mathrm{mm}^{2}$ respectively for diagnosing malignant breast cancer. This study demonstrates ADC value were same for b0 - 800, b0 - 1200, and b0 - $1600 \mathrm{~s} / \mathrm{mm}^{2}$ (Table 2). So we conclude that the use of higher b value in combination can be used in differentiating benign and malignant breast lesion at 3.0 Tesla DWI as per recent meta-analysis by Dorrius et al. [38], also stated result similar to our finding that those of $b=0$ and $1000 \mathrm{~s} / \mathrm{mm}^{2}$ are recommended for the best improvement of differentiating between benign and malignant lesions, hence instead of using multiple DWI with different $b$ values it is wise to perform this sequence as per our studied $b$ value combination, either with an intermediate or high b value. This way, the acquisition scan time will be shortened, which is comfortable for the patients and less artifacts due to movement of patient. Our study has some limitations firstly, the patients group included in this study was comparatively small, and secondly it was a retrospective study performed in our university so our study could be selection bias. Thirdly, use of only $5 \mathrm{~b}$ values in our study, so we need to include more b value range in our study. Nevertheless, further study of larger population may be needed to confirm the diagnostic efficiency of ADC in differentiating benign and malignant breast lesions.

\section{Conclusion}

The determined mean and cut-off ADC values in different studies should not be accepted as absolute values. We 
authors believe that each institution should define its own mean and cut-off ADC values for differentiating benign and malignant breast lesions.

\section{References}

[1] Gianfelice, D., Khiat, A., Amara, M., Belblidia, A. and Boulanger, Y. (2003) MR Imaging-Guided Focused US Ablation of Breast Cancer: Histopathologic Assessment of Effectiveness-Initial Experience. Radiology, 227, 849-855. http://dx.doi.org/10.1148/radiol.2281012163

[2] Kuhl, C.K., Schrading, S., Leutner, C.C., Morakkabati-Spitz, N., Wardelmann, E., Fimmers, R., et al. (2005) Mammography, Breast Ultrasound, and Magnetic Resonance Imaging for Surveillance of Women at High Familial Risk for Breast Cancer. Journal of Clinical Oncology, 23, 8469-8476. http://dx.doi.org/10.1200/JCO.2004.00.4960

[3] Leach, M.O., et al. (2005) Screening with Magnetic Resonance Imaging and Mammography of a UK Population at High Familial Risk of Breast Cancer: A Prospective Multicentre Cohort Study (MARIBS). Lancet, 365, 1769-1778. http://dx.doi.org/10.1016/S0140-6736(05)66481-1

[4] Sardanelli, F., Giuseppetti, G.M., Panizza, P., Bazzocchi, M., Fausto, A., Simonetti, G., et al. (2004) Sensitivity of MRI versus Mammography for Detecting Foci of Multifocal, Multicentric Breast Cancer in Fatty and Dense Breasts Using the Whole-Breast Pathologic Examination as a Gold Standard. AJR American Journal of Roentgenology, 183, 1149-1157. http://dx.doi.org/10.2214/ajr.183.4.1831149

[5] Wenkel, E., Geppert, C., Schulz-Wendtland, R., Uder, M., Kiefer, B., Bautz, W., et al. (2007) Diffusion Weighted Imaging in Breast MRI: Comparison of Two Different Pulse Sequences. Academic Radiology, 14, 1077-1083. http://dx.doi.org/10.1016/j.acra.2007.06.006

[6] Cai, H.M., Liu, L.Z., Peng, Y.X., Wu, Y.P. and Li, L. (2014) Diagnostic Assessment by Dynamic Contrast-Enhanced and Diffusion-Weighted Magnetic Resonance in Differentiation of Breast Lesions under Different Imaging Protocols. BMC Cancer, 14, 366. http://dx.doi.org/10.1186/1471-2407-14-366

[7] Kul, S., Cansu, A., Alhan, E., Dinc, H., Gunes, G. and Reis, A. (2011) Contribution of Diffusion-Weighted Imaging to Dynamic Contrast-Enhanced MRI in the Characterization of Breast Tumors. AJR American Journal of Roentgenology, 196, 210-217. http://dx.doi.org/10.2214/AJR.10.4258

[8] Partridge, S.C., DeMartini, W.B., Kurland, B.F., Eby, P.R., White, S.W. and Lehman, C.D. (2009) Quantitative Diffusion-Weighted Imaging as an Adjunct to Conventional Breast MRI for Improved Positive Predictive Value. AJR American Journal of Roentgenology, 193, 1716-1722. http://dx.doi.org/10.2214/AJR.08.2139

[9] Tan, S.L., Rahmat, K., Rozalli, F.I., Mohd-Shah, M.N., Aziz, Y.F.A., Yip, C.H., et al. (2014) Differentiation between Benign and Malignant Breast Lesions Using Quantitative Diffusion- Weighted Sequence on 3 T MRI. Clinical Radiology, 69, 63-71. http://dx.doi.org/10.1016/j.crad.2013.08.007

[10] Yoo, H., Shin, H.J., Baek, S., Cha, J.H., Kim, H., Chae, E.Y. and Kim, H.H. (2014) Diagnostic Performance of Apparent Diffusion Coefficient and Quantitative Kinetic Parameters for Predicting Additional Malignancy in Patients with Newly Diagnosed Breast Cancer. Magnetic Resonance Imaging, 32, 867-874. http://dx.doi.org/10.1016/j.mri.2014.04.012

[11] Koh, D.-M. and Collins, D.J. (2007) Diffusion-Weighted MRI in the Body: Applications and Challenges in Oncology. American Journal of Roentgenology, 188, 1622-1635. http://dx.doi.org/10.2214/AJR.06.1403

[12] Woodhams, R., Ramadan, S., Stanwell, P., Sakamoto, S., Hata, H., Ozaki, M., Kan, S. and Inoue, Y. (2011) Diffusion-Weighted Imaging of the Breast: Principles and Clinical Applications. RadioGraphics, 31, 1059-1084. http://dx.doi.org/10.1148/rg.314105160

[13] Guo, Y., Cai, Y.-Q., Cai, Z.-L., Gao, Y.-G., An, N.-Y., Ma, L., Mahankali, S. and Gao, J.-H. (2002) Differentiation of Clinically Benign and Malignant Breast Lesions Using Diffusion-Weighted Imaging. Journal of Magnetic Resonance Imaging, 16, 172-178. http://dx.doi.org/10.1002/jmri.10140

[14] Yoshikawa, M.I., Ohsumi, S., Sugata, S., Kataoka, M., Takashima, S., Mochizuki, T., Ikura, H. and Imai, Y. (2008) Relation between Cancer Cellularity and Apparent Diffusion Coefficient Values Using Diffusion-Weighted Magnetic Resonance Imaging in Breast Cancer. Radiation Medicine, 26, 222-226. http://dx.doi.org/10.1007/s11604-007-0218-3

[15] Park, M.J., Cha, E.S., Kang, B.J., Ihn, Y.K. and Baik, J.H. (2007) The Role of Diffusion-Weighted Imaging and the Apparent Diffusion Coefficient (ADC) Values for Breast Tumors. Korean Journal of Radiology, 8, 390-396. http://dx.doi.org/10.3348/kjr.2007.8.5.390

[16] Partridge, S.C., DeMartini, W.B., Kurland, B.F., Eby, P.R., White, S.W. and Lehman, C.D. (2010) Differential Diagnosis of Mammographically and Clinically Occult Breast Lesions on Diffusion-Weighted MRI. Journal of Magnetic Resonance Imaging, 31, 562-570. http://dx.doi.org/10.1002/jmri.22078

[17] Partridge, S.C., Mullins, C.D., Kurland, B.F., Allain, M.D., DeMartini, W.B., Eby, P.R. and Lehman, C.D. (2010) Apparent Diffusion Coefficient Values for Discriminating Benign and Malignant Breast MRI Lesions: Effects of Lesion 
Type and Size. American Journal of Roentgenology, 194, 1664-1673. http://dx.doi.org/10.2214/AJR.09.3534

[18] Kuhl, C.K., Mielcareck, P., Klaschik, S., Leutner, C., Wardelmann, E., Gieseke, J. and Schild, H.H. (1999) Dynamic Breast MR Imaging: Are Signal Intensity Time Course Data Useful for Differential Diagnosis of Enhancing Lesions? Radiology, 211, 101-110. http://dx.doi.org/10.1148/radiology.211.1.r99ap38101

[19] Kuhl, C.K., Schild, H.H. and Morakkabati, N. (2005) Dynamic Bilateral Contrast-Enhanced MR Imaging of the Breast: Trade-Off between Spatial and Temporal Resolution. Radiology, 236, 789-800. http://dx.doi.org/10.1148/radiol.2363040811

[20] Warren, R.M., Pointon, L., Thompson, D., Hoff, R., Gilbert, F.J., Padhani, A., Easton, D., Lakhani, S.R. and Leach, M.O. (2005) Reading Protocol for Dynamic Contrast-Enhanced MR Images of the Breast: Sensitivity and Specificity Analysis. Radiology, 236, 779-788. http://dx.doi.org/10.1148/radiol.2363040735

[21] Oshida, K., Nagashima, T., Ueda, T., Yagata, H., Tanabe, N., Nakano, S., Nikaidou, T., Funatsu, H., Hashimoto, H. and Miyazaki, M. (2005) Pharmacokinetic Analysis of Ductal Carcinoma in Situ of the Breast Using Dynamic MR Mammography. European Radiology, 15, 1353-1360. http://dx.doi.org/10.1007/s00330-005-2661-9

[22] Pediconi, F., Catalano, C., Occhiato, R., Venditti, F., Fraioli, F., Napoli, A., Kirchin, M.A. and Passariello, R. (2005) Breast Lesion Detection and Characterization at Contrast-Enhanced MR Mammography: Gadobenate Dimeglumine versus Gadopentetate Dimeglumine. Radiology, 237, 45-56. http://dx.doi.org/10.1148/radiol.2371041369

[23] Le Bihan, D., Turner, R., Douek, P. and Patronas, N. (1992) Diffusion MR Imaging: Clinical Applications. American Journal of Roentgenology, 159, 591-599. http://dx.doi.org/10.2214/ajr.159.3.1503032

[24] Rubesova, E., Grell, A.-S., De Maertelaer, V., Metens, T., Chao, S.-L. and Lemort, M. (2006) Quantitative Diffusion IMAGING in Breast Cancer: A Clinical Prospective Study. Journal of Magnetic Resonance Imaging, 24, 319-324. http://dx.doi.org/10.1002/jmri.20643

[25] Tsushima, Y., Takahashi-Taketomi, A. and Endo, K. (2009) Magnetic Resonance (MR) Differential Diagnosis of Breast Tumors Using Apparent Diffusion Coefficient (ADC) on 1.5-T. Journal of Magnetic Resonance Imaging, 30 , 249-255. http://dx.doi.org/10.1002/jmri.21854

[26] Yabuuchi, H., Matsuo, Y., Okafuji, T., Kamitani, T., Soeda, H., Setoguchi, T., et al. (2008) Enhanced Mass on Contrast-Enhanced Breast MR Imaging: Lesion Characterization Using Combination of Dynamic Contrast-Enhanced and Diffusion-Weighted MR Images. Journal of Magnetic Resonance Imaging, 28, 1157-1165. http://dx.doi.org/10.1002/jmri.21570

[27] Baltzer, P.A., Renz, D.M., Herrmann, K.-H., Dietzel, M., Krumbein, I., Gajda, M., Camara, O., Reichenbach, J.R. and Kaiser, W.A. (2009) Diffusion-Weighted Imaging (DWI) in MR Mammography (MRM): Clinical Comparison of Echo Planar Imaging (EPI) and Half-Fourier Single-Shot Turbo Spin Echo (HASTE) Diffusion Techniques. European Radiology, 19, 1612-1620. http://dx.doi.org/10.1007/s00330-009-1326-5

[28] Belli, P., Costantini, M., Bufi, E., Magistrelli, A., La Torre, G. and Bonomo, L. (2010) Diffusion-Weighted Imaging in Breast Lesion Evaluation. Radiation Medicine, 115, 51-69. http://dx.doi.org/10.1007/s11547-009-0430-6

[29] Chen, X., Li, W.-L., Zhang, Y.-L., Wu, Q., Guo, Y.-M. and Bai, Z.-L. (2010) Meta-Analysis of Quantitative Diffusion-Weighted MR Imaging in the Differential Diagnosis of Breast Lesions. BMC Cancer, 10, 693. http://dx.doi.org/10.1186/1471-2407-10-693

[30] Fornasa, F., Pinali, L., Gasparini, A., Toniolli, E. and Montemezzi, S. (2011) Diffusion-Weighted Magnetic Resonance Imaging in Focal Breast Lesions: Analysis of 78 Cases with Pathological Correlation. Radiation Medicine, 116, 264-275. http://dx.doi.org/10.1007/s11547-010-0602-4

[31] Hatakenaka, M., Soeda, H., Yabuuchi, H., Matsuo, Y., Kamitani, T., Oda, Y., Tsuneyoshi, M. and Honda, H. (2008) Apparent Diffusion Coefficients of Breast Tumors: Clinical Application. Magnetic Resonance in Medical Sciences, 7, 23-29. http://dx.doi.org/10.2463/mrms.7.23

[32] Kuroki-Suzuki, S., Kuroki, Y., Nasu, K., Nawano, S., Moriyama, N. and Okazaki, M. (2007) Detecting Breast Cancer with Non-Contrast MR Imaging: Combining Diffusion-Weighted and STIR Imaging. Magnetic Resonance in Medical Sciences, 6, 21-27. http://dx.doi.org/10.2463/mrms.6.21

[33] Pereira, F.P., Martins, G., Figueiredo, E., Domingues, M.N.A., Domingues, R.C., da Fonseca, L.M.B. and Gasparetto, E.L. (2009) Assessment of Breast Lesions with Diffusion-Weighted MRI: Comparing the Use of Different b Values. American Journal of Roentgenology, 193, 1030-1035. http://dx.doi.org/10.2214/AJR.09.2522

[34] Rahbar, H., Partridge, S.C., Eby, P.R., DeMartini, W.B., Gutierrez, R.L., Peacock, S. and Lehman, C.D. (2011) Characterization of Ductal Carcinoma in Situ on Diffusion Weighted Breast MRI. European Radiology, 21, 2011-2019. http://dx.doi.org/10.1007/s00330-011-2140-4

[35] Kuroki, Y., Nasu, K., Kuroki, S., Murakami, K., Hayashi, T., Sekiguchi, R. and Nawano, S. (2004) DiffusionWeighted Imaging of Breast Cancer with the Sensitivity Encoding Technique: Analysis of the Apparent Diffusion Coefficient Value. Magnetic Resonance in Medical Sciences, 3, 79-85. http://dx.doi.org/10.2463/mrms.3.79 
[36] Marini, C., Iacconi, C., Giannelli, M., Cilotti, A., Moretti, M. and Bartolozzi, C. (2007) Quantitative DiffusionWeighted MR Imaging in the Differential Diagnosis of Breast Lesion. European Radiology, 17, 2646-2655.

http://dx.doi.org/10.1007/s00330-007-0621-2

[37] Woodhams, R., Matsunaga, K., Iwabuchi, K., Kan, S., Hata, H., Kuranami, M., Watanabe, M. and Hayakawa, K. (2005) Diffusion-Weighted Imaging of Malignant Breast Tumors: The Usefulness of Apparent Diffusion Coefficient (ADC) Value and ADC Map for the Detection of Malignant Breast Tumors and Evaluation of Cancer Extension. Journal of Computer Assisted Tomography, 29, 644-649. http://dx.doi.org/10.1097/01.rct.0000171913.74086.1b

[38] Dorrius, M.D., Dijkstra, H., Oudkerk, M. and Sijens, P.E. (2014) Effect of b Value and Pre-Admission of Contrast on Diagnostic Accuracy of 1.5-T Breast DWI: A Systematic Review and Meta-Analysis. European Radiology, 24, 2835-2847. http://dx.doi.org/10.1007/s00330-014-3338-z 